\title{
Pharmacophore-based virtual screening and density functional theory approach to identifying novel butyrylcholinesterase inhibitors
}

\author{
Sugunadevi SAKKIAH, Keun Woo LEE*
}

Division of Applied Life Science (BK21 Program), Systems and Synthetic Agrobiotech Center (SSAC), Plant Molecular Biology and Biotechnology Research Center (PMBBRC), Research Institute of Natural Science (RINS), Gyeongsang National University (GNU), Jinju 660-701, Korea

Aim: To identify the critical chemical features, with reliable geometric constraints, that contributes to the inhibition of butyrylcholinesterase (BChE) function.

Methods: Ligand-based pharmacophore modeling was used to identify the critical chemical features of BChE inhibitors. The generated pharmacophore model was validated using various techniques, such as Fischer's randomization method, test set, and decoy set. The best pharmacophore model was used as a query in virtual screening to identify novel scaffolds that inhibit BChE. Compounds selected by the best hypothesis in the virtual screening were tested for drug-like properties, and molecular docking study was applied to determine the optimal orientation of the hit compounds in the BChE active site. To find the reactivity of the hit compounds, frontier orbital analysis was carried out using density functional theory.

Results: Based on its correlation coefficient (0.96), root mean square (RMS) deviation (1.01), and total cost (105.72), the quantitative hypothesis Hypo 1 consisting of $2 \mathrm{HBA}, 1 \mathrm{Hy}$-Ali, and $1 \mathrm{Hy}$-Ar was selected as the best hypothesis. Thus, Hypo 1 was used as a 3D query in virtual screening of the Maybridge and Chembridge databases. The hit compounds were filtered using ADMET, Lipinski's Rule of Five, and molecular docking to reduce the number of false positive results. Finally, 33 compounds were selected based on their critical interactions with the significant amino acids in BChE's active site. To confirm the inhibitors' potencies, the orbital energies, such as HOMO and LUMO, of the hit compounds and 7 training set compounds were calculated. Among the 33 hit compounds, 10 compounds with the highest HOMO values were selected, and this set was further culled to 5 compounds based on their energy gaps important for stability and energy transfer. From the overall results, 5 hit compounds were confirmed to be potential BChE inhibitors that satisfied all the pharmacophoric features in Hypo1.

Conclusion: This study pinpoints important chemical features with geometric constraints that contribute to the inhibition of BChE activity. Five compounds are selected as the best hit BchE-inhibitory compounds.

Keywords: cholinesterase; butyrylcholinesterase; Alzheimer's disease; virtual screening; density functional theory; molecular docking; ligand-based pharmacophore modeling theory

Acta Pharmacologica Sinica (2012) 33: 964-978; doi: 10.1038/aps.2012.21; published online 11 Jun 2012

\section{Introduction}

Cholinesterases (ChEs) are involved in the degradation of choline and show similarity in protein sequence but differences in their kinetic properties. On the basis of their substrate and inhibitor specificities, cholinesterases are divided into two subfamilies: acetylcholinesterases (AChEs; EC 3.1.1.7) and butyrylcholinesterases (BChEs; EC 3.1.1.8). AChE is predominantly present in the central and peripheral nervous system,

\footnotetext{
* To whom correspondence should be addressed.

E-mail kwlee@gnu.ac.kr

Received 2012-01-03 Accepted 2012-02-22
}

as well as in muscles. In muscles, AChE terminates impulse transmission by the rapid hydrolysis of acetylcholine to acetic acid and choline ${ }^{[1]}$. BChE is primarily synthesized in the liver and secreted into plasma, and it is responsible for the hydrolysis of a variety of choline (hydrophilic and hydrophobic) and non-choline esters ${ }^{[2]}$. BChE plays a key role in cholinergic synapses by terminating acetylcholine action, although the complete physiological function of BChE remains unclear ${ }^{[3]}$. Both cholinesterase enzymes belong to the super family of $\mathrm{a} / \beta$-hydrolase fold proteins ${ }^{[4]}$. Both $\mathrm{AChE}$ and BChE exist as multimers of catalytic subunits in globular forms such as G1, G2, and G4 that contain one, two, or four subunits, respec- 
tively. The hydrolysis of substrates by both enzymes proceeds through a transacylation step involving nucleophilic and general acid-base elements ${ }^{[5]}$. BChE acts as a scavenger protein that protects the cholinergic system against anticholinesterase poisons. BChE is the sole carboxylesterase ${ }^{[6,7]}$ with recognized toxicological and pharmacological importance in scavenging and detoxification of numerous ester-containing drugs, pro$\operatorname{drugs}^{[8,9]}$, and poisonous carbamyl- and phosphoryl-esters, including nerve agents ${ }^{[10,11]}$.

Currently, BChE is emerging as an important pharmacological target in Alzheimer's disease (AD) therapy ${ }^{[12]}$. A 40\%-90\% increase in $\mathrm{BChE}$ expression and activity have been found in AD brain neuronal plaques ${ }^{[13]}$. BChE is capable of compensating for reduced AChE catalytic functions in the synaptic cleft $^{[14,15]}$ and shows significantly increased activity $(30 \%-60 \%)$ during the time course of $\mathrm{AD}^{[16,17]}$. Hence, in recent years, many scientists and researchers have shown keen interest in designing small molecules that can inhibit BChE activity ${ }^{[18-23]}$. However, there is also increasing evidence of BChE's involvement in non-cholinergic functions such as cell differentiation $^{[24]}$, neurogenesis, and the formation of amyloid plaques in $\mathrm{AD}^{[25-27]}$.

In this work, we used computer-aided drug design approaches to identify novel and potent inhibitors of BChE. Pharmacophore studies are more cost-effective than experimental chemical screening of large databases. A 3D pharmacophore model was generated for BChE based on a series of well-known inhibitors. The best quantitative model was used as a 3D query for virtual screening of chemical databases to discover novel hit compounds. The virtual screening results revealed a small subset of database compounds that were promising potential hit compounds for BChE inhibition. The hits were subsequently filtered by Lipinski's Rule of Five, ADME (absorption, distribution, metabolism, and excretion) properties, and molecular docking. Finally, density functional theory (DFT) was used to calculate the orbital energy value and energy gap for the molecules screened by docking.

\section{Computational methods}

Pharmacophore modeling is one of the most frequently used and valuable methods to discover novel scaffolds for various targets.

\section{Selection of compounds}

To construct the BChE data set, 71 compounds were collected with their corresponding reported inhibitory activity values $\left(\mathrm{IC}_{50}\right)$ which were tested using the same bioassay technique from various publications ${ }^{[28-32]}$. The BChE data set was divided into two sets: training and test sets that contained 26 and 45 compounds, respectively. The training set was prepared based on the following criteria: (i) a minimum of 16 diverse compounds were selected to avoid any chance correlation; (ii) the activity data should have a range of 4-5 orders of magnitude; (iii) the compounds should be selected to provide clear, concise information to avoid redundancy or bias in terms of both structural features and activity range; (iv) the most active compounds should be included so that they provide information on the most critical features required for a reliable/rational pharmacophore model; and (v) the inclusion of any compound known to be inactive due to steric hindrance must be avoided. The training set was used to build the quantitative hypothesis based on principles of structural diversity and $\mathrm{IC}_{50}$ values that spanned a wide activity range, from $3.6 \mathrm{nmol} / \mathrm{L}$ to $11000 \mathrm{nmol} / \mathrm{L}$ (Figure 1). The test set was used to evaluate the predictive ability of the generated pharmacophore model. Both the training and test set compounds were classified into three categories based on their activity values. The compounds with $\mathrm{IC}_{50}$ values less than or equal to $100 \mathrm{nmol} / \mathrm{L}$ were considered to be highly active $(+++)$, compounds with an activity range between $100 \mathrm{nmol} / \mathrm{L}$ and $10000 \mathrm{nmol} / \mathrm{L}$ were considered to be moderately active $(++)$, and compounds with $\mathrm{IC}_{50}$ values greater than or equal to $10000 \mathrm{nmol} / \mathrm{L}$ were set as low activity compounds $(+)$. The $2 \mathrm{D}$ structures of the training and test set molecules were drawn using ChemSketch ${ }^{[24]}$, and the structures were converted into their corresponding $3 \mathrm{D}$ form using DS.

\section{Pharmacophore modeling}

Quantitative hypotheses were generated, and the best hypothesis was selected based on the models' ability to predict the biological activity of novel compounds from various chemical databases using Discovery Studio v2.5.5 (DS, www.accelrys. com, San Diego, CA, USA). There are generally two methods to generate molecular conformation: FAST and BEST. The FAST algorithm only considers existing conformers and interrupts a search as soon as a pharmacophore matching conformation is found, whereas the BEST algorithm additionally "tweaks" bond distances, angles, and dihedral angles of pregenerated conformers on the fly to achieve the best matches. Herein, we used the BEST conformation method to generate multiple acceptable conformations for each compound present in the training and test sets with $20 \mathrm{kcal} / \mathrm{mol}$ as the energy cutoff $^{[33]}$. All default parameters were used to generate the pharmacophore, except the uncertainty default value (3.0) was changed to $2.0^{[34]}$. The uncertainty is the ratio of the reported activity value relative to the minimum, and the maximum values must be greater than 1.0. The uncertainty value affects the categorization of ligands in the data set as either active or inactive compounds and is used during the constructive and subtractive phases. Here, an uncertainty value of 2.0 was more suitable for our data set because the compound activities spanned the requisite 4 orders of magnitude; this choice has been confirmed by evidence in the literature ${ }^{[35,36]}$. The feature mapping/DS protocol was used to identify common features present in the active inhibitors of BChE. This protocol computes a maximum of 1000 possible pharmacophore features mappings for the selected ligands. The selected features from the feature mapping were used as one of the key inputs for the 3D-QSAR pharmacophore generation module using a HypoGen algorithm. The HypoGen algorithm further estimates the activity of each training set compound by computing regres- 


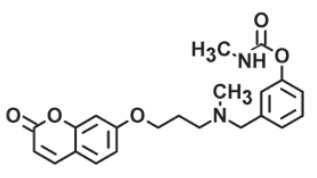

Compound 1 (3.6)

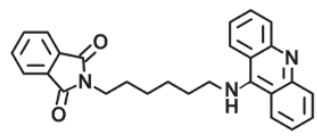

Compound 2 (7.8)

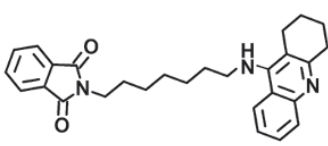

Compound $3(10)$

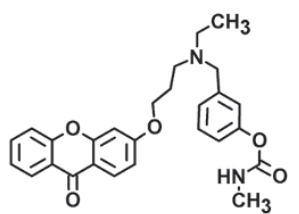

Compound 4 (21)

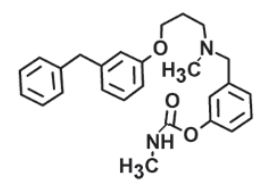

Compound 5 (22.1)

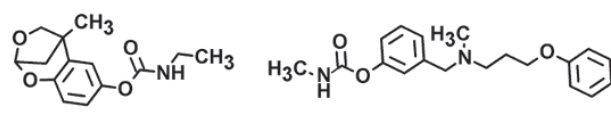

Compound 6 (23)

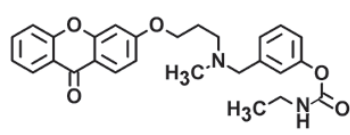

Compound 8 (26)

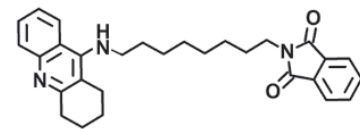

Compound 9 (29)

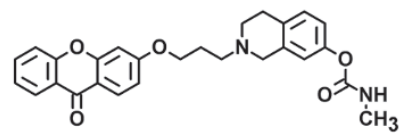

Compound 10 (34.1)

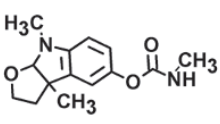

Compound 11 (56)

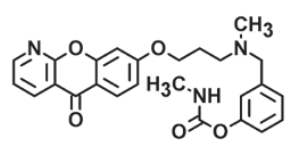

Compound 12 (76)

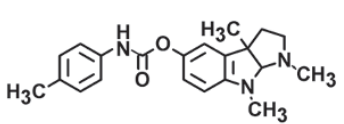

Compound 13 (250)

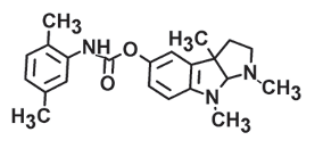

Compound 14 (490)

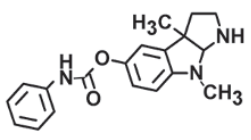

Compound 15 (612)

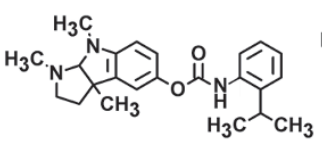

Compound 16 (650)

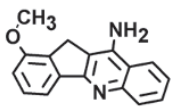

Compound 22 (3000)

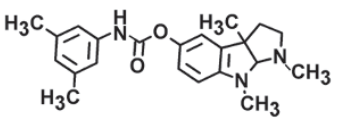

Compound 17 (798)

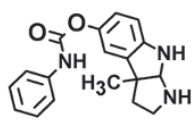

Compound 18 (897)

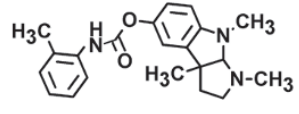

Compound 19 (1180)

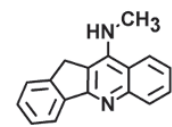

Compound 20 (1600)

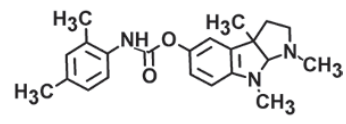

Compound 21 (1820)

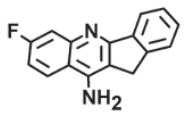

Compound 24 (5700)

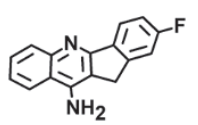

Compound 25 (7100)

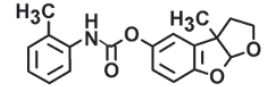

Compound 26 (11400)

Figure 1. Set of 26 chemically diverse compounds used as the training set in 3D-QSAR discovery studio/pharmacophore generation. IC ${ }_{50}$ values, in $\mathrm{nmol} / \mathrm{L}$, are indicated in parentheses for each compound.

sion analysis using parameters such as the relationship of geometric fit value versus the negative logarithm of the activity. While generating the quantitative model, a minimum of 0 to a maximum of 5 features were selected to build a series of hypotheses. Ten quantitative pharmacophore models were generated with corresponding statistical parameters such as cost values, root mean square (RMS), and fit values. The best quality hypothesis was selected based on cost values as defined by Debnath's methods ${ }^{[34]}$.

\section{Hypothesis validation}

In general, pharmacophore models should be statistically significant, accurately predict the activity of molecules, and retrieve active compounds from databases. The best pharmacophore model was validated using various potent approaches such as Fischer's randomization, test set, and decoy set ${ }^{[33]}$. The main purpose of validating a quantitative pharmacophore model is to determine its capacity to identify active compounds, as well as its predictive ability for corresponding molecules. Fischer's randomization test was performed simultaneously during the original hypotheses generation and produced a number of random spreadsheets depending on the selected significance level (90\%, 95\%, 98\%, and 99\%) by shuffling the activity values present in the training set. Here, a 95\% significance level was selected. Nineteen random spreadsheets were produced by randomly shuffling the activity value of the training set compounds, and the test generated hypotheses using the same chemical features and parameters used to develop the original hypothesis. Test and decoy sets were used to check whether the best hypothesis was able to select molecules with orders of magnitude of activity similar to that of the active training set and to determine how well the model hypothesis could differentiate potential BChE inhibitors from other compounds, respectively. The test set consisted of structurally diverse chemical compounds from the training set to ascertain the broadness of pharmacophore predictability. The decoy set was prepared by calculating the 1D property of 25 active inhibitors of BChE and 2075 inactive or unknown molecules. EF and GF were calculated using the following equations: 


$$
\begin{aligned}
& \mathrm{EF}=\left(\mathrm{H}_{\mathrm{a}} / \mathrm{H}_{\mathrm{t}}\right) /(\mathrm{A} / \mathrm{D}) \\
& \mathrm{GF}=\left(\mathrm{H}_{\mathrm{a}} / 4 \mathrm{H}_{\mathrm{t}} \mathrm{A}\right)(3 \mathrm{~A}+\mathrm{Ht}) *\left\{1-\left[\left(\mathrm{H}_{\mathrm{t}}-\mathrm{H}_{\mathrm{a}}\right) /(\mathrm{D}-\mathrm{A})\right]\right\}
\end{aligned}
$$

where $\mathrm{H}_{\mathrm{a}}$ is the total number of active compounds in the hit list, $\mathrm{H}_{\mathrm{t}}$ is the number of hits retrieved from the database, $\mathrm{A}$ is the total number of active compounds in the database, and D is the total number of molecules in the database.

\section{Virtual screening}

Pharmacophore-based database searching was used to find potential hit compounds that could repress or trigger $\mathrm{BChE}$ activity. The generated, well-validated hypothesis was used as a 3D structural query in the virtual screening of databases such as Maybridge and Chembridge to retrieve novel scaffolds for BChE inhibition. The Fast Flexible search method from Ligand Pharmacophore Mapping/DS was applied to retrieve hits that satisfy the chemical moiety requirements and spatially map with corresponding features in the pharmacophoric query $^{[37]}$.

\section{Drug likeness filtration}

Poor pharmacokinetic properties are one of the main causes for the termination of a compound's entry or progression along the drug development pipeline. The medicinal chemist needs compounds with good pharmacokinetic properties; thus, all of the hit compounds obtained from database searching were filtered by applying ADME and the Rule of Five developed by Lipinski ${ }^{[38]}$. To obtain compounds with good pharmacokinetic properties, ADMET descriptors were calculated. ADMET was applied to check whether the compounds are able to cross the blood-brain barrier (BBB) and have good solubility, human intestinal absorption (HIA), and low toxicity. Here, we mainly focused on oral bioavailability, low or no hepatotoxicity, and the capacity to penetrate the BBB, which is a key decision filter for central nervous system drug discovery. The compounds that satisfied the abovementioned properties were selected for molecular docking studies. Lipinski's rule of 5 states that $\log P \leq 5$, molecular weight $\leq 500$, and number of hydrogen bond acceptors $\leq 10$ and donors $\leq 5$. Compounds violating more than one of these rules may have problems with bioavailability, therefore these parameters were calculated by DS to eliminate compounds that did not pass the above criterias.

\section{Molecular docking}

Molecular docking generates a score for each compound based on the binding affinities of protein-ligand complexes. Molecular docking was used to identify the small molecules that were able to fit well into the binding site of BChE proteins. LigandFit ${ }^{[39]}$ was used to execute the molecular docking studies and to determine the accurate orientation of ligands in protein active sites. The LigandFit module was classified into three stages: (i) docking, when an attempt was made to dock a ligand into a user defined binding site; (ii) in situ ligand minimization; and (iii) scoring, when various scoring functions were calculated for each pose of the ligands. The 3D crystal structure of BChE (PDB code: 1P0I) was downloaded from Protein Data Bank
(PDB, www.rcsb.org) with good resolution $(2 \AA)$. The protein was prepared by adding the hydrogen atoms by applying CHARMm force field ${ }^{[40]}$ using the Molecular simulation module. After protein preparation, the active site of the protein must be identified before docking the small molecules. The active site of the protein can be represented as a binding site, essentially as a set of points on a grid that lie in a cavity. Two methods were applied to define the binding site for the protein: (i) based on the receptor shape using "eraser" algorithm and (ii) volume occupied by the known ligand position already in an active site. For this study, we preferred the second method to find the active cavity of BChE. Initially, the docking parameters were validated by docking the co-crystal molecule into the active site of BChE. The hit molecules from the virtual screening process and 5 active inhibitors were docked in the active site of BChE to find the most suitable orientation and compound binding ability. During the docking process, the top 10 conformations were generated for each ligand based on the docking score after energy minimization using the smart minimizer method, which begins with the steepest descent method followed by the conjugate gradient method. The docked poses were validated by the hydrogen bond interactions between the candidate molecules and active site residues.

\section{Density functional theory}

The main aim of the orbital energies calculation was to provide valuable information about the electrostatic properties of the BChE inhibitors. DFT is a successful and promising approach adopted by quantum chemists in the quantum mechanical simulation of periodic systems ${ }^{[41]}$. There is substantial evidence that DFT provides an accurate description of the electronic and structural properties of small molecules by computing the electronic structure of matter. The selected docked poses of the hit compounds from the molecular docking studies were used as input for the DFT instead of the compounds' bioactive conformations. Because the docking results showed the suitable binding orientation of hit compounds, it was suitable for calculating the orbital energies such as $\mathrm{HOMO}$ and LUMO using DS. Calculating the orbital energy using B3LYP provided information regarding the capacity of the molecules to transfer their energies from a HOMO, which can act as an electron donor, to a LUMO, which can act as an electron acceptor. These electrostatic property calculations could provide useful information for designing novel BChE inhibitors.

\section{Results and discussion}

A ligand-based pharmacophore method was used to elucidate the spatial arrangement of chemical features that were crucial for the interaction of structurally diverse and potent BChE inhibitors with their target protein. Ligand-based approaches reveal the important and common chemical features of diverse ligands, and these features can then be used as 3D query in virtual screenings of large chemical databases to identify novel hit compounds. 


\section{Pharmacophore model}

The HypoGen algorithm was used to construct quantitative hypotheses that correlated the experimental and the predicted activity values of the inhibitors. At the end of each run, the top ten hypotheses were generated based on a set of 26 chemically diverse inhibitors of BChE (Figure 1), and the statistical parameters values such as cost, correlation $(r)$, and RMS for each hypothesis are shown in Table 1.

Among the ten hypotheses, nine hypotheses contained 1 hydrogen bond acceptor (HBA) and 1 hydrophobic aliphatic (Hy-Ali) group, which indicates that these chemical features are necessary for BChE inhibition. Out of the 10 hypotheses, only 3 hypotheses were selected for further processing based on the maximum fit value (greater than 9). Debnath's analy$\operatorname{sis}^{[42]}$, used to select the best hypothesis, states that the best pharmacophore model should have the highest cost difference, good correlation coefficient, least RMS, and lowest total cost values. Cost differences represent the difference between the null and total cost of hypothesis. A 40-60 bit difference leads to a predictive correlation probability of $75 \%-90 \%$, and if the difference is greater than 60 bits, the hypothesis is assumed to have a correlation probability of greater than $90 \%{ }^{[31]}$. Hypo1 showed the highest cost difference of 120.12 bits, compared with Hypo4 and Hypo5, indicating its significance. The correlation coefficient is based on linear regression derived from the geometric fit index; Hypo1 showed the highest correlation coefficient (0.96), demonstrating its high predictive ability. The RMS factor represents the deviation of the predicted activity value from the experimental value, and the RMS values were 1.02, 1.23, and 1.24 for Hypo1, Hypo4, and Hypo5, respectively. This result also supports the conclusion that Hypo1 was significant when compared with the two other hypotheses. The reliability of a pharmacophore model also depends on whether the total cost value is distant from the null cost and close to the fixed cost. The fixed cost represents a simple model that fits all data perfectly, while the null cost presumes that there is no relationship in the data and that the experimental activities are normally distributed around their average value. The fixed and total cost values of Hypo1 were 94.82 and 108.57, respectively. Thus, Hypo1, which consisted of $2 \mathrm{HBA}, 1 \mathrm{Hy}-\mathrm{Ali}$, and 1 hydrophobic aromatic (Hy-Ar), was selected as the best hypothesis and was employed for further analyses. The chemical features and 3D spatial arrangement of Hypo1 are depicted in Figure 2.

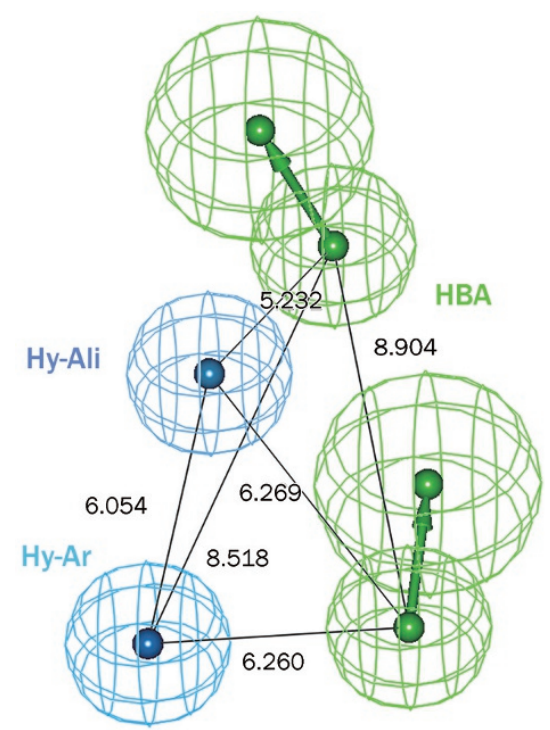

Figure 2. The Hypo1 pharmacophore model and its geometric parameters, where HBA: hydrogen bond acceptor; Hy-Ali: hydrophobic aliphatic; and Hy-Ar: hydrophobic aromatic.

Hypo1 was used to estimate the inhibitory activities of 26 training set compounds to elucidate its predictive accuracy. Hypo1 was able to predict the inhibitory activity value of the 26 training set compounds in the same order of magnitude (Table 2). One moderately active and two inactive compounds

Table 1. Information of statistical significance and predictive power presented in cost values measured in bits for the top 10 hypotheses as a result of automated 3D QSAR pharmacophore generation.

\begin{tabular}{|c|c|c|c|c|c|c|}
\hline Hypo № & Total cost & Cost difference $^{a}$ & $\mathrm{RMS}^{\mathrm{b}}$ & Correlation & Features $^{b}$ & Max fit \\
\hline Hypo1 & 105.72 & 120.12 & 1.01 & 0.96 & $2 \mathrm{HBA}, 1 \mathrm{Hy}-\mathrm{Ali}, 1 \mathrm{Hy}-\mathrm{Ar}$ & 8.58 \\
\hline Нypo2 & 103.93 & 116.91 & 1.10 & 0.95 & 2 HBA, 1 Hy-Ali, 1 RA & 9.54 \\
\hline Нуро3 & 109.97 & 115.87 & 1.15 & 0.94 & 2 HBA, 1 Hy-Ali, 1 Hy-Ar & 8.85 \\
\hline Hypo4 & 112.57 & 113.27 & 1.23 & 0.93 & 2 HBA, 1 Hy-Ali, 1 RA & 9.18 \\
\hline Hypo5 & 112.98 & 112.86 & 1.24 & 0.93 & 2 HBA, 1 Hy-Ali, 1 RA & 9.48 \\
\hline Нypo8 & 117.43 & 108.41 & 1.39 & 0.91 & 2 HBA, 1 Hy-Ali, 1 Hy-Ar & 7.86 \\
\hline Hypo9 & 118.78 & 107.06 & 1.42 & 0.91 & 2 HBA, 1 Hy-Ali, 1 RA & 7.50 \\
\hline Hypo10 & 118.82 & 107.02 & 1.43 & 0.91 & $2 \mathrm{HBA}, 2 \mathrm{Hy}-\mathrm{Ar}$ & 7.86 \\
\hline
\end{tabular}

${ }^{a}$ Cost difference between the null and the total cost. The null cost, the fixed cost and the configuration cost are 225.84, 92.399 and 15.79 , respectively.

${ }^{\mathrm{b}}$ Abbreviation used for features: RMS, root mean square deviation; HBA, hydrogen bond acceptor; Hy-Ali, hydrophobic aliphatic, Hy-Ar, hydrophobic aromatic and RA, ring aromatic. 
Table 2. Actual and estimated activity of the training set molecules based on the pharmacophore model Hypo1.

\begin{tabular}{|c|c|c|c|c|c|c|}
\hline $\begin{array}{c}\text { Compound } \\
\text { № }\end{array}$ & $\begin{array}{c}\text { Fit } \\
\text { value }^{a}\end{array}$ & $\begin{array}{l}\text { Exp IC }{ }_{50} \\
\mathrm{nmol} / \mathrm{L}\end{array}$ & $\begin{array}{l}\text { Pred } \mathrm{IC}_{50} \\
\mathrm{nmol} / \mathrm{L}\end{array}$ & Error $^{b}$ & $\begin{array}{l}\text { Exp } \\
\text { scale }^{c}\end{array}$ & $\begin{array}{l}\text { Pred } \\
\text { scale }^{c}\end{array}$ \\
\hline 1 & 6.89 & 3.6 & 5.5 & +1.5 & +++ & +++ \\
\hline 2 & 6.38 & 7.8 & 18 & +2.3 & +++ & +++ \\
\hline 3 & 6.28 & 10 & 22 & +2.2 & +++ & +++ \\
\hline 4 & 6.36 & 21 & 19 & -1.1 & +++ & +++ \\
\hline 5 & 6.22 & 22 & 26 & +1.2 & +++ & +++ \\
\hline 6 & 6.08 & 23 & 36 & +1.6 & +++ & +++ \\
\hline 7 & 6.11 & 25 & 33 & +1.3 & +++ & +++ \\
\hline 8 & 6.31 & 26 & 21 & -1.2 & +++ & +++ \\
\hline 9 & 6.39 & 29 & 18 & -1.7 & +++ & +++ \\
\hline 10 & 6.12 & 34 & 33 & -1.0 & +++ & +++ \\
\hline 11 & 6.16 & 56 & 30 & -1.9 & +++ & +++ \\
\hline 12 & 6.3 & 76 & 21 & -3.5 & +++ & +++ \\
\hline 13 & 4.85 & 250 & 600 & +2.4 & ++ & ++ \\
\hline 14 & 4.75 & 490 & 760 & +1.5 & ++ & ++ \\
\hline 15 & 4.25 & 610 & 2400 & +3.9 & ++ & + \\
\hline 16 & 4.85 & 650 & 600 & -1.1 & ++ & ++ \\
\hline 17 & 4.62 & 800 & 1000 & +1.3 & ++ & ++ \\
\hline 18 & 4.9 & 900 & 540 & -1.7 & ++ & ++ \\
\hline 19 & 4.55 & 1200 & 1200 & +1.0 & + & + \\
\hline 20 & 4.01 & 1600 & 4200 & +2.6 & + & + \\
\hline 21 & 4.72 & 1800 & 810 & -2.2 & + & ++ \\
\hline 22 & 3.94 & 1800 & 810 & -2.2 & + & ++ \\
\hline 22 & 3.94 & 3000 & 5000 & +1.7 & + & + \\
\hline 23 & 4.29 & 3900 & 2200 & -1.8 & + & + \\
\hline 24 & 4.01 & 5700 & 4200 & -1.3 & + & + \\
\hline 25 & 4.09 & 7100 & 3500 & -2.1 & + & + \\
\hline 26 & 4.28 & 11000 & 2200 & -5.1 & + & + \\
\hline
\end{tabular}

${ }^{\text {a }}$ Fit value indicates how well the features in the pharmacophore overlap the chemical features in the molecule.

${ }^{\mathrm{b}}$ Division of higher value of experimental or predicted $\mathrm{IC}_{50}$ by lower predicted or experimental $I \mathrm{IC}_{50}$ value. ' + ' indicates that the predicted $\mathrm{IC}_{50}$ is higher than the experimental I $\mathrm{I}_{50}$; ' - ' indicates that the predicted $I \mathrm{I}_{50}$ is lower than the experimental $\mathrm{IC}_{50}$; a value of 1 indicates that the predicted $I C_{50}$ is equal to the experimental $I C_{50}$.

${ }^{c}$ Activity scale: $I C_{50}<100 \mathrm{nmol} / \mathrm{L}=+++$ (highly active); $100 \mathrm{nmol} / \mathrm{L} \leq$ $\mathrm{IC}_{50}<1000 \mathrm{nmol} / \mathrm{L}=++$ (moderately active); $I \mathrm{C}_{50} \geq 10000 \mathrm{nmol} / \mathrm{L}=+$ (low active).

were underestimated and overestimated as inactive and moderately active, respectively. All of the active compounds were predicted in their own activity ranges, indicating the predictive ability of Hypo1. Hypo1 aligned with the most active compound $1\left(\mathrm{IC}_{50}: 3.6 \mathrm{nmol} / \mathrm{L}\right)$ and least active compound $26\left(\mathrm{IC}_{50}: 11400 \mathrm{nmol} / \mathrm{L}\right.$ ) in the training set (Figure 3). From this analysis, we suggest that Hypo1 was able to estimate the activity of compounds to a high degree of accuracy relative to their experimental $\mathrm{IC}_{50}$ values (Table 2). The error value was defined as the ratio between experimental and predicted activity value, and Hypo1 demonstrated remarkable consistency. The best pharmacophore model, Hypo1, was validated by various methods such as Fisher's randomization, a test set, and a decoy set to demonstrate its robustness and
A

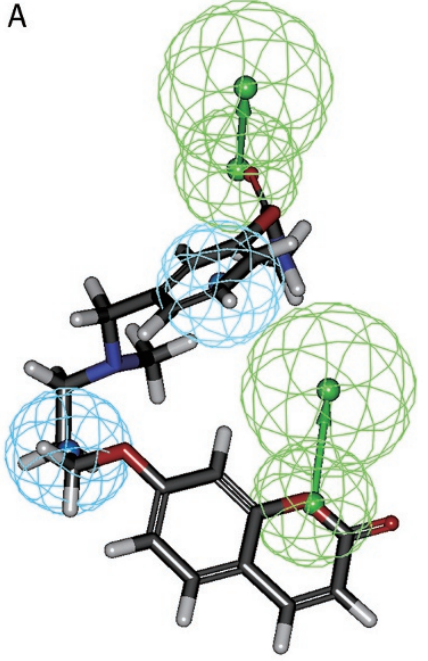

B

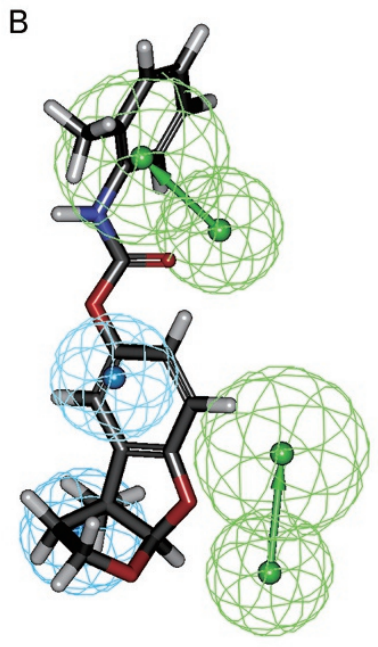

Figure 3. The best pharmacophore model Hypo1 aligned to training set compounds: $\mathrm{A}$ ) active compound $1\left(\mathrm{IC}_{50} 3.6 \mathrm{nmol} / \mathrm{L}\right)$ and $\left.\mathrm{B}\right)$ low activity compound $26\left(\mathrm{IC}_{50} 11400 \mathrm{nmol} / \mathrm{L}\right)$. The pharmacophore features are color coded (HBA, hydrogen bond acceptor: green; Hy-Ali, hydrophobic aliphatic: light cyan; Hy-Ar, hydrophobic aromatic: cyan).

statistical significance.

\section{Validation of the pharmacophore model} Fischer's randomization test

Fischer's test was applied to evaluate the significance of Hypo1 based on statistical validation. A confidence level of $95 \%$ was chosen, and a total of 19 random spreadsheets were generated to produce the hypothesis. The significance of the hypothesis was calculated using the formula $S=[1-(1+X) /$ $Y] \times 100$, where $X$ is the total number of hypotheses having a total cost lower than the original hypothesis, and $\mathrm{Y}$ is the total number of HypoGen runs (initial+random runs). Here, $X=0$ and $Y=(1+19)$, hence $95 \%=\{1-[(1+0) /(19+1)]\} \times 100$. The total cost of 19 random pharmacophore models compared with Hypo1 showed that the original hypothesis was far superior to the 19 other hypotheses, which indicated that the Hypo1 was not generated by chance (Figure 4). This result provided confidence that the Hypo1 could be a best hypothesis that contains all the necessary chemical features to inhibit BChE activity.

\section{Test set validation}

The test set contains 45 structurally distinct compounds from training set molecules. The test set was used to examine the ability of Hypo1 to predict the activity of external compounds in the same activity range. Except for one active compound that was underestimated as moderately active, all of the remaining compounds are predicted on their own activity range by Hypo1 (Table 3). Hypo1 shows the strong correlation coefficient of 0.94 between experimental and predicted BChE inhibitory activity values for the test set (Figure 5). This result also showed that Hypo1 fit not only for the training set compounds but also for the external compounds; this result 


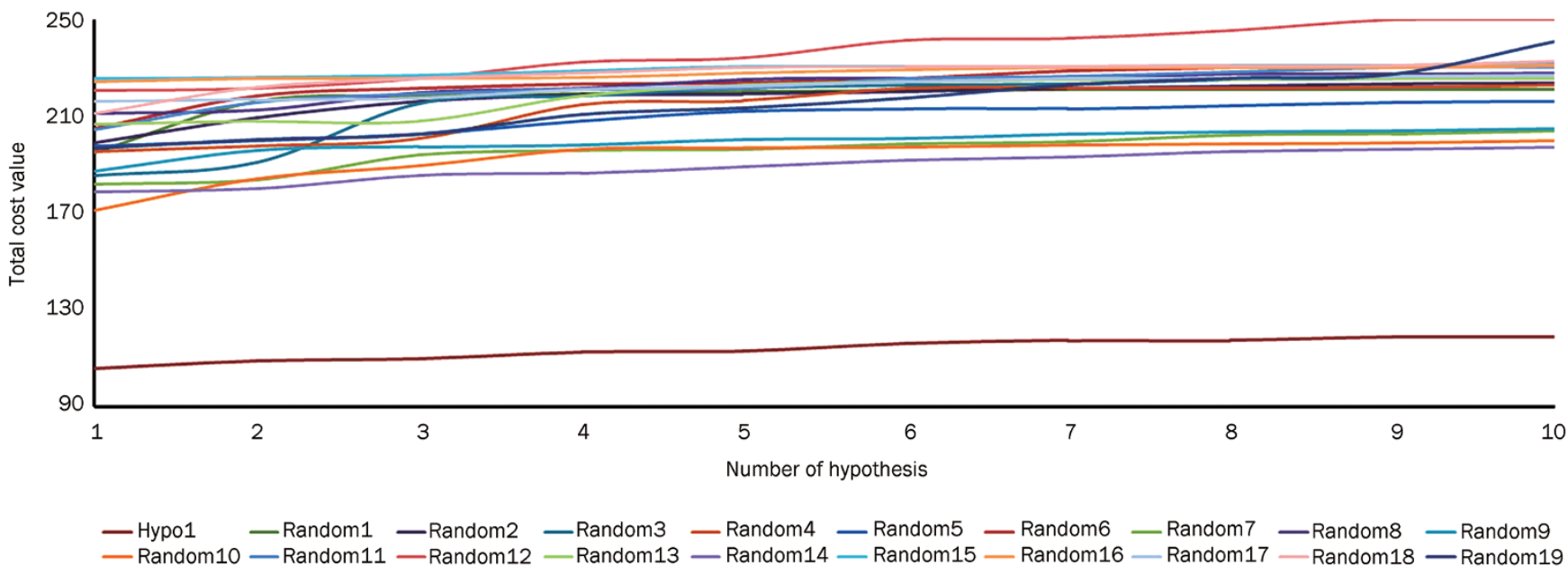

Figure 4. The difference in cost between 19 scrambled runs. The $95 \%$ confidence level was selected.

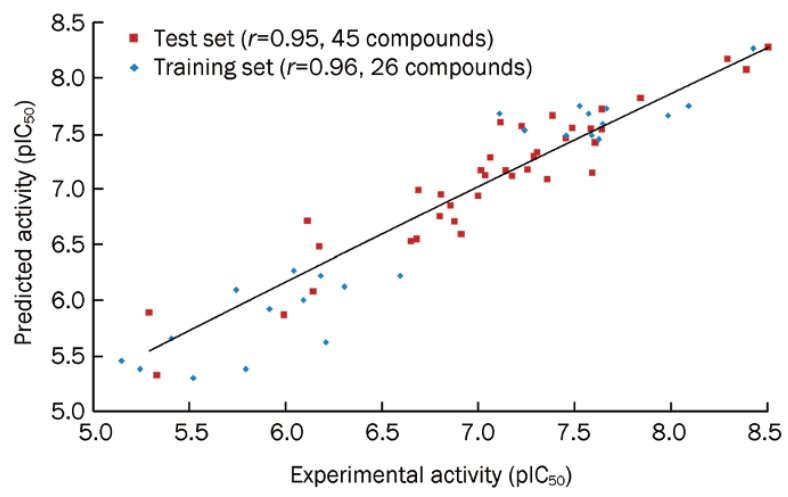

Figure 5. Plot of the correlation between the experimental activity and the activity predicted by Hypo1 for the test set molecules (in brown) and training set molecules (in blue).

also demonstrated the predictive ability of Hypo1 to differentiate the active and inactive BChE inhibitors.

\section{Decoy set validation}

As a final validation, decoy set screening was performed using the Best Flexible searching module/DS. To determine the robustness of Hypo1, four parameters were calculated: false positives, false negatives, enrichment factor (EF), and goodness of fit score (GF). EF and GF were calculated using the following set of parameters: hit lists $\left(\mathrm{H}_{\mathrm{t}}\right)$, number of active percent yields $(\% \mathrm{Y})$, percent ratio of actives in the hit lists $(\% \mathrm{~A})$, false negatives, and false positives (Table 4). Hypo1 succeeded in the retrieval of $76 \%$ of the active compounds from the decoy set. It predicted 6 active compounds to be inactive compounds (false negatives). Hypo1 showed a GH score of 0.86 , indicating that Hypo1 had a greater tendency to show true positives. On the basis of the overall validations, we were strongly assured that the Hypo1 demonstrated excellent prediction of BChE inhibitor activities.

\section{Virtual screening}

The validated hypothesis, Hypo1, was used as a 3D structural query for retrieving novel candidate molecules from the Maybridge (60000) and Chembridge (50000) chemical databases. A total of 45496 hit compounds were obtained from the first screening; among these, 1619 compounds were selected for further analysis by applying maximum fit value of greater than 8. Subsequently, these molecules were tested for ADMET and Lipinski's Rule of Five. ADMET properties calculated the values of blood-brain barrier (BBB) penetration, solubility, cytochrome P450 (CYP450) 2D6 inhibition, hepatotoxicity, HIA, plasma protein binding (PPB), and assessed a broad range of ligand toxicity. The drug should not cross the BBB, hence the level ' 3 ' was selected to represent low penetration of BBB. The value of 0 in CYP26 and hepatotoxicity indicates that the molecules are of low toxicity. The cutoff values of solubility and HIA were 3 and 0, respectively. Out of the 1619 molecules, 202 molecules passed the BBB level, absorption, solubility, and toxicity criteria. These hit compounds were subjected to further filtering by applying Lipinski's Rule of Five, which states that LogP should be less than 5, the molecular weight less than 500, the number of hydrogen bond donors less than 5, the number of hydrogen bond acceptors less than 10 , and the number of rotatable bonds less than 10 . The flexibility of the molecules and the total number of hydrogen bond acceptors and hydrogen bond donors are important predictors that a compound will have good oral bioavailability. Ultimately, 84 molecules were selected as hits based on druglike properties. The hit compounds from the virtual screening process were subjected to molecular docking to reduce the false positive rate.

\section{Molecular docking}

A BChE complex with butanoic acid from PDB was chosen as the target protein for molecular docking. The establishment and reorganization of specific covalent or non-covalent 
Table 3. Experimental and predicted $I \mathrm{I}_{50}$ values of 45 test set molecules against Hypo1.

\begin{tabular}{|c|c|c|c|c|c|c|c|}
\hline $\begin{array}{c}\text { Compound } \\
\text { № }\end{array}$ & Structure & Fit value & $\begin{array}{l}\operatorname{Exp~IC}_{50} \\
(\mathrm{nmol} / \mathrm{L})\end{array}$ & $\begin{array}{l}\text { Pred } \mathrm{IC}_{50} \\
(\mathrm{nmol} / \mathrm{L})\end{array}$ & Error $^{\mathrm{a}}$ & $\begin{array}{l}\text { Exp } \\
\text { Scale }^{b}\end{array}$ & $\begin{array}{c}\text { Pred } \\
\text { Scale }^{b}\end{array}$ \\
\hline 1 & & 6.90 & 3.0 & 5.34 & +1.78 & +++ & +++ \\
\hline 2 & & 6.36 & 3.3 & 8.51 & +2.58 & +++ & +++ \\
\hline 3 & & 6.36 & 3.9 & 8.51 & +2.18 & +++ & +++ \\
\hline 4 & & 6.07 & 4.9 & 6.85 & +1.40 & +++ & +++ \\
\hline 5 & & 6.07 & 5.0 & 6.85 & +1.37 & +++ & +++ \\
\hline 6 & & 5.93 & 13.9 & 15.31 & +1.10 & +++ & +++ \\
\hline 7 & & 5.35 & 22.1 & 19.34 & -1.14 & +++ & +++ \\
\hline 8 & & 6.05 & 24.0 & 38.57 & +1.61 & +++ & +++ \\
\hline 9 & & 5.73 & 24.5 & 29.42 & 1.20 & +++ & +++ \\
\hline 10 & & 5.77 & 24.8 & 72.32 & +2.92 & +++ & +++ \\
\hline 11 & & 6.69 & 25.2 & 28.84 & +1.14 & +++ & +++ \\
\hline 12 & & 6.69 & 28.0 & 28.84 & +1.03 & +++ & +++ \\
\hline 13 & & 5.17 & 31.0 & 29.62 & -1.05 & +++ & +++ \\
\hline 14 & & 5.17 & 31.6 & 28.62 & -1.10 & +++ & +++ \\
\hline 15 & & 7.08 & 34.1 & 35.36 & +1.04 & +++ & +++ \\
\hline
\end{tabular}


nog

www.nature.com/aps

Sakkiah S et al

972

Compound

№

16

17

18

19

20

21

22

23

24

25

26

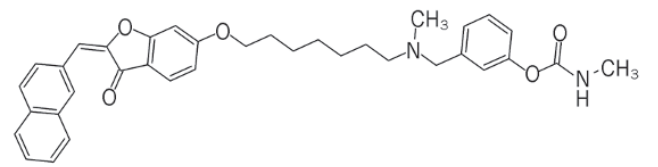

27

$\begin{array}{llllll}\text { Fit value } & \begin{array}{c}\text { Exp IC }_{50} \\ (\mathrm{nmol} / \mathrm{L})\end{array} & \begin{array}{l}\text { Pred IC }_{50} \\ (\mathrm{nmol} / \mathrm{L})\end{array} & \text { Error }^{\mathrm{a}} & \text { Exp } & \text { Pred } \\ \text { Scale }^{\mathrm{b}} & \text { Scale }^{\mathrm{b}}\end{array}$

40.0

22.05

5.71

42.6

82.64

5.71

44.3

$82.64+1.87$

5.96

48.3

47.38

$-1.02$

5.92

50.0

5.80

54.0

67.56

$+1.25$

5.96

54.6

47.38

$-1.15$

6.19

57.9

27.49

$-2.11$

$5.75 \quad 65.0$

76.99

$-1.18$

5.79

70.3

68.81

$-1.02$

5.28

74.8

25.39

$-2.95$

+++ $\quad+++$

$5.91 \quad 84.4$

52.36

$-1.61$

(to be continued)

Acta Pharmacologica Sinica 


\begin{tabular}{|c|c|c|c|c|c|c|c|}
\hline $\begin{array}{c}\text { Compound } \\
\text { № }\end{array}$ & Structure & Fit value & $\begin{array}{l}\operatorname{Exp~IC}_{50} \\
(\mathrm{nmol} / \mathrm{L})\end{array}$ & $\begin{array}{l}\text { Pred IC } C_{50} \\
(\mathrm{nmol} / \mathrm{L})\end{array}$ & Error ${ }^{a}$ & $\begin{array}{l}\text { Exp } \\
\text { Scale }\end{array}$ & $\begin{array}{r}\text { Pred } \\
\text { Scale }\end{array}$ \\
\hline 28 & & 5.75 & 90 & 75.87 & -1.19 & +++ & +++ \\
\hline 29 & & 5.30 & 98 & 115.96 & +1.18 & +++ & ++ \\
\hline 30 & & 5.22 & 120 & 257.32 & +2.14 & ++ & ++ \\
\hline 31 & & 4.93 & 130 & 198.69 & +1.53 & ++ & ++ \\
\hline 32 & & 5.10 & 136 & 142.40 & +1.05 & ++ & ++ \\
\hline 33 & & 5.58 & 153 & 113.40 & -1.35 & ++ & ++ \\
\hline 34 & & 5.74 & 155 & 178.64 & +1.15 & ++ & ++ \\
\hline 35 & & 5.62 & 200 & 103.15 & -1.94 & ++ & ++ \\
\hline 36 & & 5.70 & 205 & 284.84 & +1.39 & ++ & ++ \\
\hline 37 & & 5.16 & 220 & 299.54 & +1.36 & ++ & ++ \\
\hline 38 & & 5.70 & 237 & 244.84 & +1.03 & ++ & ++ \\
\hline 39 & & 5.11 & 660 & 330.68 & -2 & ++ & ++ \\
\hline
\end{tabular}




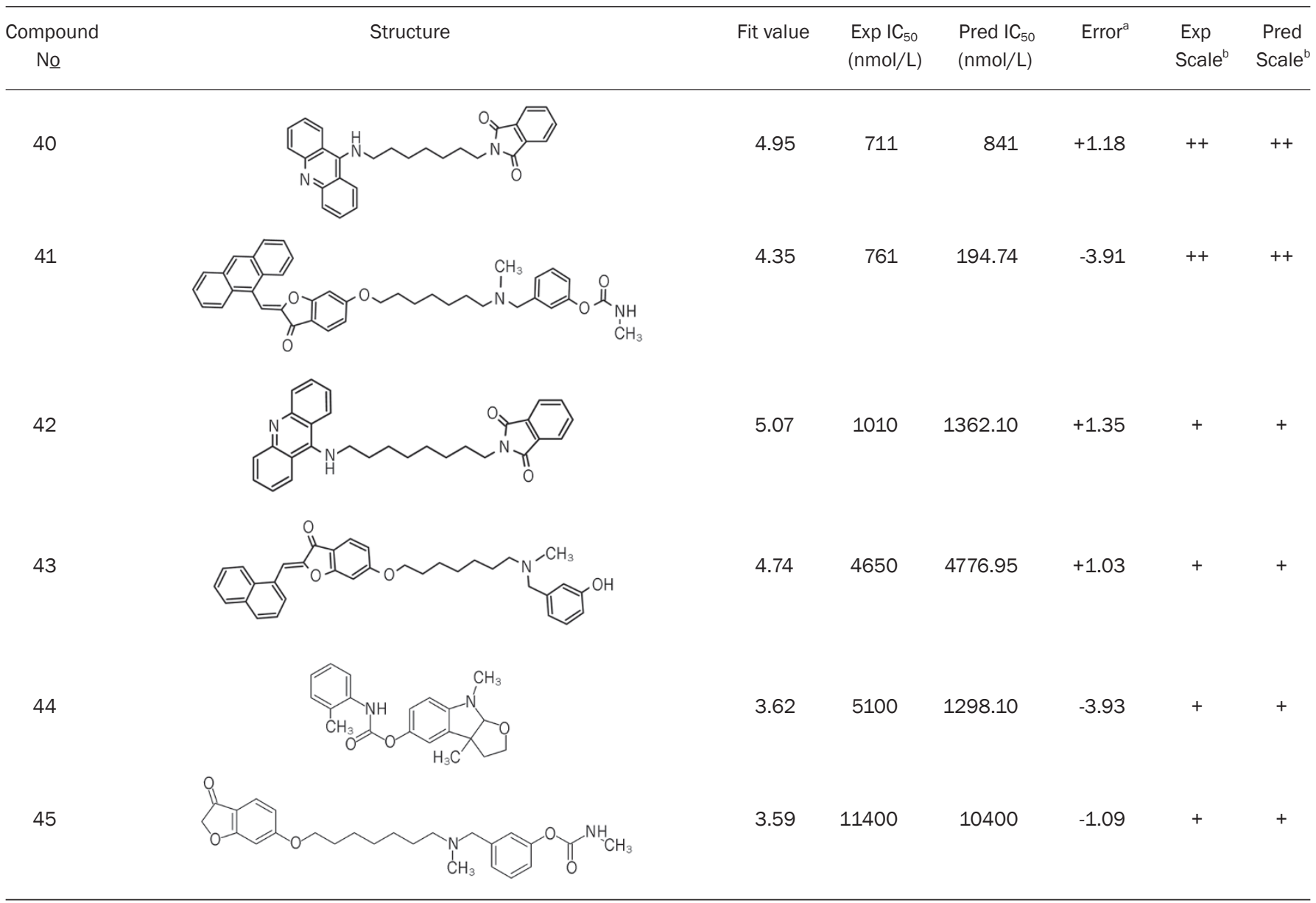

a ' + ' indicates that the predicted $I \mathrm{I}_{50}$ is higher than the experimental $I \mathrm{I}_{50}$; ' '-' indicates that the predicted $I \mathrm{C}_{50}$ is lower than the experimental $I \mathrm{C}_{50}$; $a$ value of 1 indicates that the predicted $I C_{50}$ is equal to the experimental $I C_{50}$.

${ }^{\mathrm{b}}$ Activity scale : $\mathrm{IC}_{50}<100 \mathrm{nmol} / \mathrm{L}=+++$ (highly active); $100 \mathrm{nmol} / \mathrm{L} \leq \mathrm{IC}_{50}<1000 \mathrm{nmol} / \mathrm{L}=++$ (moderately active); $\mathrm{IC} \mathrm{C}_{50} \geq 1000 \mathrm{nmol} / \mathrm{L}=+($ low active).

Table 4. Statistical parameter from screening test set molecules.

\begin{tabular}{rlc}
\hline No & \multicolumn{1}{c}{ Parameter } & Values \\
\hline 1 & Total number of molecules in database $(\mathrm{D})$ & 2100 \\
2 & Total number of actives in database $(\mathrm{A})$ & 25 \\
3 & Total number of hit molecules from the database $(\mathrm{Ht})$ & 21 \\
4 & Total number of active molecules in hit list $(\mathrm{Ha})$ & 19 \\
5 & \% Yield of active $[(\mathrm{Ha} / \mathrm{Ht}) \times 100]$ & 90.47 \\
6 & \% Ratio of actives $[(\mathrm{Ha} / \mathrm{A}) \times 100]$ & 76 \\
7 & Enrichment Factor $(\mathrm{EF})$ & 76 \\
8 & False negatives $(\mathrm{A}-\mathrm{Ha})$ & 6 \\
9 & False Positives $\left(\mathrm{H}_{\mathrm{t}}-\mathrm{H}_{\mathrm{a}}\right)$ & 2 \\
10 & Goodness of fit score $(\mathrm{GF})$ & 0.87
\end{tabular}

interactions between substrates or inhibitors play a crucial role in biological function. Three distinct domains in the active site confer selectivity of BChE inhibitors. The first domain is an acyl binding pocket that contains two residues (L286 and V288) responsible for the binding of larger substrates with acyl groups ${ }^{[43]}$. A second domain is found near the lip of the active site cavity, and a third domain is defined as the choline binding site (or cation-pi site). The catalytic domain of BChE is composed of nucleophilic serine, histidine, and glutamate residues. The substrate was stabilized between the oxyanion hole and acyl binding pocket of the "catalytic triad" composed of S198, E197, and H438 of the active esteratic site. The mechanism of catalysis depends on the charge relay system, in which the imidazole ring of H438 relays electrons from E197 to S198 and causes the hydroxyl oxygen of S198 to become a nucleophile $^{[44]}$. A nucleophilic attack of this hydroxyl oxygen on the ester bond of the substrate leads to an acyl-enzyme intermediate and a free choline moiety. Then, the acyl group is hydrolyzed from S198 by the nucleophilic attack of a water molecule that is activated by taking a proton from $\mathrm{H} 438$ to form a catalytic triad.

Initially the co-crystal was docked in the active site of BChE to check whether the selected parameters are able to produce the most suitable binding orientation. The RMSD value of $0.79 \AA$ was obtained when the best docked pose overlapped with the co-crystal, which revealed that the default parameters 
are valid to find the best orientation of BChE in the active site. The same parameters were therefore employed to dock the candidate compounds. The selected candidate molecules from the virtual screening were docked in the BChE active site. The top-ranked 84 compounds based on the docking score were selected as the best potential inhibitors and were manually validated for critical interaction with vital amino acids in the active site of BChE. Intermolecular hydrogen bonding was observed between active residues S198, E197, and H438 in the active site of BChE (Figure 6). From the 84 compounds, 33 candidate molecules showed hydrogen bond interactions with S198 and H438, as well as reliable hydrophobic interactions with Y323 and F329.

\section{Density functional theory}

The orbital energies such as HOMO (highest occupied molecular orbital) and LUMO (lowest unoccupied molecular orbital) were calculated for 7 ( 5 active, one moderate and one inactive inhibitor) training set compounds and for the 33 hits from molecular docking. HOMO and LUMO are responsible for the charge transfer in a chemical reaction ${ }^{[45]}$. The calculated orbital energies of the 33 hits and known inhibitors were compared to analyze the energy transfer and stability of small molecules in protein active site. Comparing the HOMO energy with the activity value of known BChE inhibitors shows an inverse correlation, indicating that the HOMO energy of the inhibitor may transfer its electrons to some critical residues in the active site of BChE. The highest energy value of the HOMO in the hit compounds implied the greatest likelihood of strong inhibition of BChE. Hence, correlation of the HOMO values of hits and the training set molecules showed that 10 hit compounds possessed greater values than the reported inhibitors (training set) of BChE (Table 5). A smaller energy gap (between the LUMO and HOMO) of the hit molecules illustrates that the molecule are more reactive ${ }^{[46]}$. The wide energy gap in the hit
Table 5. Orbital energy value of hit leads and training set compounds.

\begin{tabular}{|c|c|c|c|c|}
\hline Name & HOMO & LUMO & $\begin{array}{c}\Delta \mathrm{E} \\
(\mathrm{eV})\end{array}$ & $\begin{array}{c}\mathrm{IC}_{50} \\
(\mathrm{nmol} / \mathrm{L})\end{array}$ \\
\hline SPB_07954 & -8.31 & -0.99 & 7.31 & \\
\hline Compound_Number_30238 & -8.32 & -0.23 & 8.08 & \\
\hline RJC_03502 & -8.55 & -0.44 & 8.11 & \\
\hline Compound_Number_14811 & -8.62 & -0.46 & 8.16 & \\
\hline BTB_07807 & -8.65 & -0.96 & 7.69 & \\
\hline Compound_Number_30080 & -8.65 & -1.19 & 7.46 & \\
\hline KM_03101 & -8.69 & -0.67 & 8.01 & \\
\hline KM_02281 & -8.70 & -0.94 & 7.76 & \\
\hline Compound_Number_15687 & -8.76 & -0.33 & 8.43 & \\
\hline Compound_Number_23227 & -8.76 & -0.97 & 7.79 & \\
\hline Training 1 & -8.77 & -0.89 & 7.89 & 3.6 \\
\hline Training 4 & -8.80 & -0.70 & 8.09 & 21 \\
\hline Training 5 & -8.80 & -0.10 & 8.70 & 22 \\
\hline Training 7 & -8.92 & -0.22 & 8.69 & 24.8 \\
\hline Training 8 & -8.98 & -0.78 & 8.21 & 26 \\
\hline Training 17 & -9.18 & -0.34 & 8.84 & 800 \\
\hline Training 26 & -9.12 & -0.28 & 8.83 & 11400 \\
\hline
\end{tabular}

molecules is unfavorable for the electron to be excited from the HOMO to the LUMO, which consequently leads to a weak affinity of the inhibitor for BChE. Among the 10 compounds, 5 hit compounds (Figure 7) were selected based on their lowest energy gaps that suggested the molecules would be reactive. Table 5 clearly showed that the moderate and inactive compounds had high energy gaps that were not suitable for the reactivity of the molecules. The atomic orbital composition of the frontier molecular orbital for compound 30080 is shown in Figure 8 . On the basis of the results above, we suggest that the hit compounds may possess equivalent or greater electronic properties compared with most active compounds and could
A

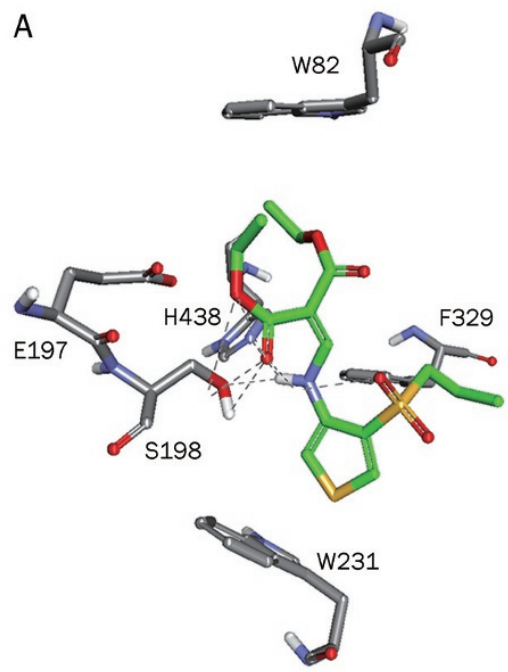

B
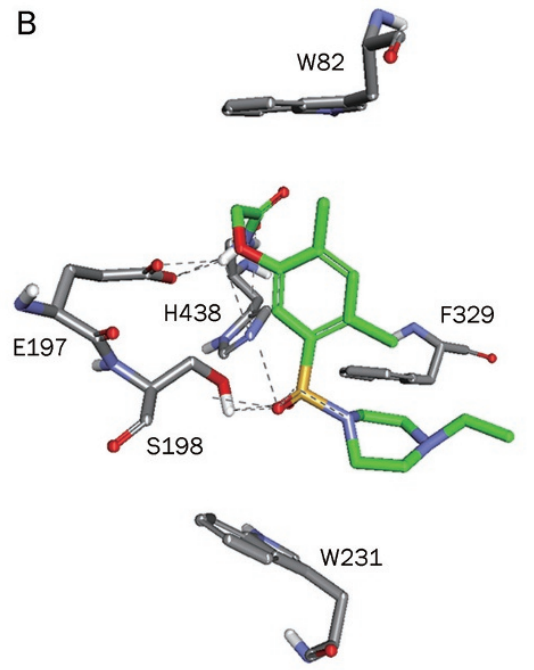

C
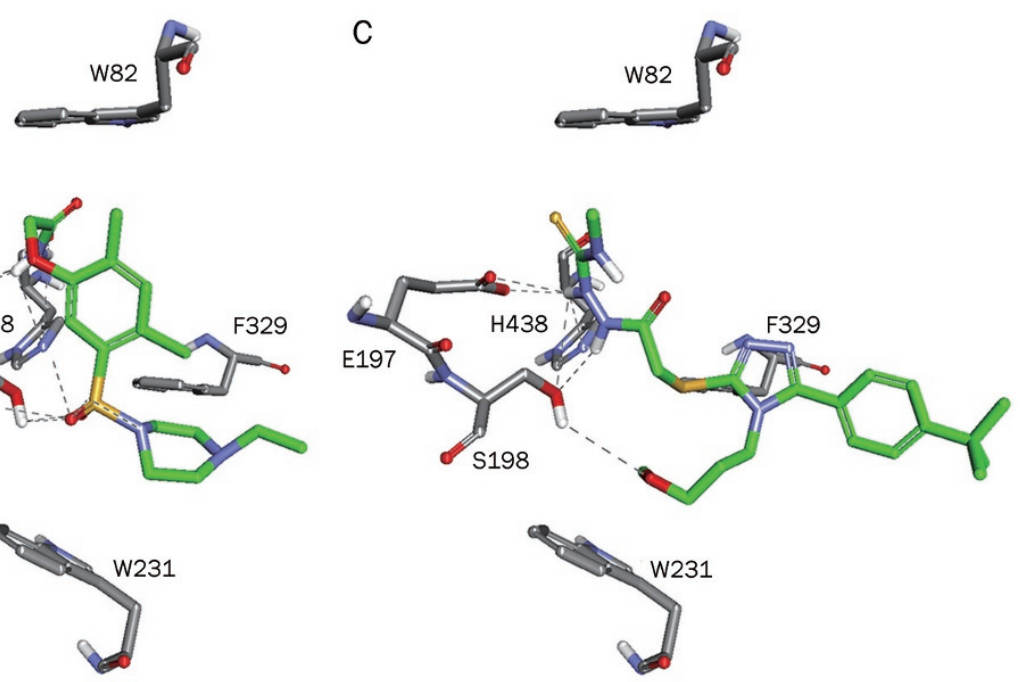

Figure 6. (A) Chembridge (compound 30080), (B) Maybridge (KM_02281), and (C) Maybridge (SPB_07954). The candidate compounds are represented as green sticks. Hydrogen bonds are shown in black. For the clarity of the docked view the Y323 was not shown. 


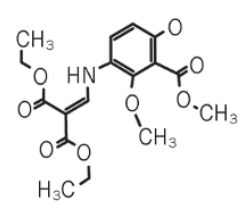

SPB_07954

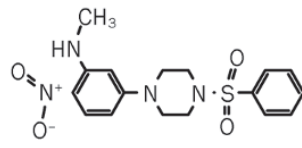

BTB_07807

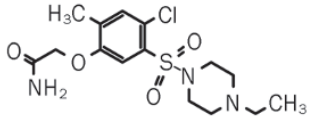

Compound_23227

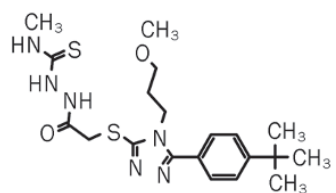

KM_02281

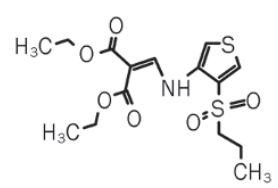

Compound_30080

Figure 7. A 2D representation of the final 5 hit compounds.

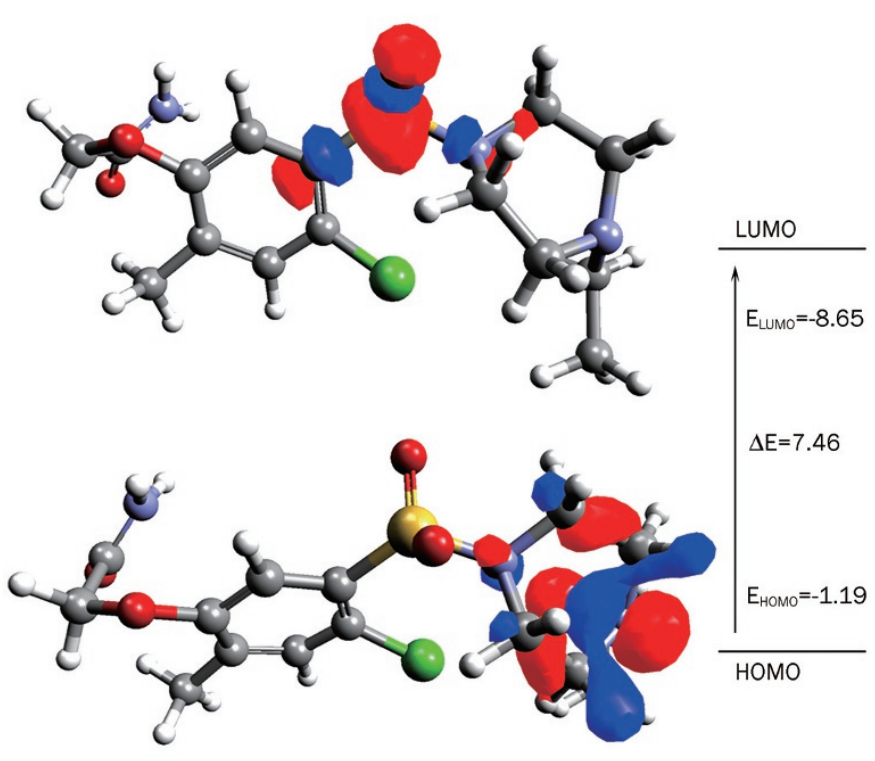

Figure 8. The orbital energy values and energy gap for compound 30080.

be used to design novel classes of BChE inhibitors.

\section{Conclusions}

In this study, pharmacophore models were generated based on a series of known BChE inhibitors. The main purpose of quantitative pharmacophore generation is to predict or differentiate the active inhibitors from inactive compounds. The best pharmacophore model, Hypo1, consisting of 2 HBA, $1 \mathrm{Hy}-\mathrm{Ali}$, and 1 Hy-Ar, was validated by Fischer's randomization method, a test set, and decoy set. Fischer's method confirmed the 95\% statistical confidence of Hypo1; the test set showed a fairly high correlation between experimental and predicted $\mathrm{IC}_{50}$ values (correlation coefficient of 0.96), indicating satisfactory predictive ability; additionally, good EF (0.76) and GF (0.87) values for Hypo1 were calculated from the decoy set. The three validation methods confirmed that Hypo1 was the best hypothesis to differentiate the active inhibitors from inactive inhibitors of BChE. Thus, Hypo1 was used as a 3D query to screen molecular structural libraries, including the Maybridge and Chembridge databases. The hit compounds were filtered using ADMET, Lipinski's Rule of Five, and molecular docking to reduce the number of false positive results. Finally, 33 compounds were selected based on their critical interactions with the significant amino acids in BChE's active site. To confirm the inhibitors' potencies, we calculated the orbital energies, such as HOMO and LUMO, for hit compounds and 7 training set compounds. From among the 33 hit compounds, 10 compounds with the highest $\mathrm{HOMO}$ values were selected, and this set was further culled to 5 compounds based on their energy gaps, which is important for stability and energy transfer. From the overall results, we confirmed that 5 hit compounds satisfied all the pharmacophoric features in Hypo1 and are potential BChE inhibitors.

\section{Acknowledgements}

This research was supported by the Basic Science Research Program (2009-0073267), the Pioneer Research Center Program (2009-0081539), and the Management of Climate Change Program (2010-0029084) through the National Research Foundation of Korea (NRF) funded by the Ministry of Education, Science, and Technology (MEST) of the Republic of Korea. This work was also supported by the Next-Generation BioGreen21 Program (PJ008038) from the Rural Development Administration (RDA) of the Republic of Korea.

\section{Author contribution}

Sugunadevi SAKKIAH contributed to all parts of the work, including experimental design, conduct, and analysis, and preparation of the manuscript. Prof Keun Woo LEE analyzed the results.

\section{References}

1 Massoulié J, Sussman J, Bon S, Silman I. Chapter 15: Structure and functions of acetylcholinesterase and butyrylcholinesterase. In: Cuello AC editor. Progress in Brain Research: Elsevier; 1993. p 13946.

2 Sun H, El Yazal J, Lockridge O, Schopfer LM, Brimijoin S, Pang YP. Predicted michaelis-menten complexes of cocaine-butyrylcholinesterase. J Biol Chem 2001; 276: 9330-6.

3 Massoulié J, Pezzementi L, Bon S, Krejci E, Vallette FM. Molecular and cellular biology of cholinesterases. Prog Neurobiol 1993; 41: 31-91.

4 Valle AM, Radić Z, Rana BK, Whitfield JB, O'Connor DT, Martin NG, et al. The cholinesterases: Analysis by pharmacogenomics in man. Chem Biol Interact 2008; 175: 343-45.

5 Quinn DM. Acetylcholinesterase: enzyme structure, reaction dynamics, and virtual transition states. Chem Rev 1987; 87: 955-79.

6 Li B, Sedlacek M, Manoharan I, Boopathy R, Duysen EG, Masson P, et al. Butyrylcholinesterase, paraoxonase, and albumin esterase, but not carboxylesterase, are present in human plasma. Biochem Pharmacol 2005; 70: 1673-84. 
7 Satoh T, Hosokawa M. Structure, function and regulation of carboxylesterases. Chem Biol Interact 2006; 162: 195-211.

8 Duysen EG, Li B, Darvesh S, Lockridge O. Sensitivity of butyrylcholinesterase knockout mice to (-)-huperzine A and donepezil suggests humans with butyrylcholinesterase deficiency may not tolerate these Alzheimer's disease drugs and indicates butyrylcholinesterase function in neurotransmission. Toxicology 2007; 233: 60-69.

9 Gardiner Sj, Begg EJ. Pharmacogenetics, drug-metabolizing enzymes, and clinical practice. Pharm Rev 2006; 58: 521-90.

10 Doctor BP, Saxena A. Bioscavengers for the protection of humans against organophosphate toxicity. Chem Biol Interact 2005; 157 158: $167-71$.

11 Masson P, Froment MT, Gillon E, Nachon F, Darvesh S, Schopfer LM. Kinetic analysis of butyrylcholinesterase-catalyzed hydrolysis of acetanilides. Biochim Biophys Acta 2007; 1774: 1139-47.

12 Vitorovic-Todorovic MD, Juranic IO, Mandic LM, Drakulic BJ. 4-Aryl-4oxo-N-phenyl-2-aminylbutyramides as acetyl- and butyrylcholinesterase inhibitors. Preparation, anticholinesterase activity, docking study, and 3D structure-activity relationship based on molecular interaction fields. Bioorg Med Chem 2010; 18: 1181-93.

13 Burgen AS. The mechanism of action of anticholinesterase drugs. Pharmac Ther 1979; 6: 579-628.

14 Mesulam MM, Guillozet A, Shaw P, Levey A, Duysen EG, Lockridge O. Acetylcholinesterase knockouts establish central cholinergic pathways and can use butyrylcholinesterase to hydrolyze acetylcholine. Neuroscience 2002; 110: 627-39.

15 Li B, Stribley JA, Ticu A, Xie W, Schopfer LM, Hammond P, et al. Abundant tissue butyrylcholinesterase and its possible function in the acetylcholinesterase knockout mouse. J Neurochem 2000; 75 : 1320-31.

16 Jhee SS, Shiovitz T, Hartman RD, Messina J, Anand R, Sramek J, Cutler $\mathrm{NR}$, et al. Centrally acting antiemetics mitigate nausea and vomiting in patients with Alzheimer's disease who receive rivastigmine. Neuropharmacology 2002; 25: 122.

17 Perry EK, Perry RH, Blessed G, Tomlinson BE. Changes in brain cholinesterases in senile dementia of Alzheimer type. Neuropathol Appl Neurobiol 1978; 4: 273-7.

18 Geula C, Nagykery N. Butyrylcholinesterase activity in the rat forebrain and upper brainstem: postnatal development and adult distribution. Exp Neurol 2007; 204: 640-57.

19 Mehrani H. Protective effect of polyurethane immobilized human butyrylcholinesterase against parathion inhalation in rat. Environmental Toxicol Pharmacol 2004; 16: 179-85.

20 Schumacher I, Arad A, Margalit R. Butyrylcholinesterase formulated in liposomes. Biotechnol Appl Biochem 1999; 30: 225-30.

21 Lockridge O, Bartels CF, Vaughan TA, Wong CK, Norton SE, Johnson LL. Complete amino acid sequence of human serum cholinesterase. J Biol Chem 1987; 262: 549-57.

22 Nicolet Y, Lockridge O, Masson P, Fontecilla-Camps, Nachon F. Crystal structure of human butyrylcholinesterase and of its complexes with substrate and products. J Biol Chem 2003; 278: 41141-7.

23 Altamirano CV, Lockridge 0 . Association of tetramers of human butyrylcholinesterase is mediated by conserved aromatic residues of the carboxy terminus. Chem Biol Interac 1999; 120: 53-60.

24 Mack A, Robitzki A. The key role of butyrylcholinesterase during neurogenesis and neural disorders: an antisense-5' butyrylcholinesterase-DNA study. Prog Neurobiol 2000; 60: 607-28.

25 Darvesh S, Hopkins DA, Geula C. Neurobiology of butyrylcholinesterase [10.1038/nrn1035]. Nat Rev Neurosci 2003; 4: 131-8.

26 Guillozet Al, Smiley JF, Mash DC, Mesulam MM. Butyrylcholinesterase in the life cycle of amyloid plaques. Ann Neurol 1997; 42: 909-18.

27 Giacobini E. Cholinesterases: New roles in brain function and in Alzheimer's disease. Neurochem Res 2003; 28: 515-22.

28 Bisi A, Belluti F, Gobbi S, Valenti P, Andrisano V, Cavrini V, et al. Acetylcholinesterase inhibitors for potential use in Alzheimer's disease: molecular modeling, synthesis and kinetic evaluation of $11 \mathrm{H}$-indeno[1,2-b]-quinolin-10-ylamine derivatives. Bioorg Med Chem 2000; 8: 497-506.

29 Wadkins RM, Tsurkan L, Hicks LD, Hatfield MJ, Edwards CC, Ross CR $2 n d$, et al. Planarity and constraint of the carbonyl groups in 1,2-diones are determinants for selective inhibition of human carboxylesterase 1. J Med Chem 2007; 50: 5727-34.

30 Moak T, Hatfield MJ, Tsurkan L, Edwards CC, Wierdl M, Danks MK, et al. Selective inhibition of carboxylesterases by isatins, indole-2,3diones. J Med Chem 2007; 50: 1876-85.

31 Luo W, Yu QS, Kulkarni SS, Parrish DA, Holloway HW, Tweedie D, et al. Inhibition of human acetyl- and butyrylcholinesterase by novel carbamates of (-)- and (+)-tetrahydrofurobenzofuran and methanobenzodioxepine. J Med Chem 2006; 49: 2174-85.

32 Fernandez-Bachiller MI, Perez C, Hernandez-Ledesma B, Bartolome $B$, Novel tacrine-melatonin hybrids as dual-acting drugs for Alzheimer disease, with improved acetylcholinesterase inhibitory and antioxidant properties. J Med Chem 2006; 49: 459-62.

33 Sakkiah S, Thangapandian S, John S, Kwon YJ, Lee KW. 3D QSAR pharmacophore based virtual screening and molecular docking for identification of potential HSP90 inhibitors. Eur J Med Chem 2010; 45: 2132-40.

34 Debnath AK. Pharmacophore mapping of a series of 2,4-diamino-5deazapteridine inhibitors of mycobacterium avium complex dihydrofolate reductase. J Med Chem 2002; 45: 41-53.

35 Kansal N, Silakari O, Ravikumar M. Three dimensional pharmacophore modelling for c-kit receptor tyrosine kinase inhibitors. Eur J Med Chem 2010; 45: 393-404.

36 Brogi S, Kladi M, Vagias C, Papazafiri P, Roussis V, Tafi A. Pharmacophore modeling for qualitative prediction of antiestrogenic activity. J Chem Inf Model 2009; 49: 2489-97.

37 Sakkiah S, Thangapandian S, John S, Lee KW. Identification of critical chemical features for aurora kinase-B inhibitors using Hip-Hop, virtual screening and molecular docking. J Mol Struct 2011; 985: 14-26.

38 Lipinski A, Lombardo F, Dominy BW, Feeney PJ. Experimental and computational approaches to estimate solubility and permeability in drug discovery and development settings. Adv Drug Delivery Rev 1997; 23: 3-25.

39 Venkatachalam CM, Jiang X, Oldfield T, Waldan M, LigandFit $n$. LigandFit: a novel method for the shape-directed rapid docking of ligands to protein active sites. J Mol Graphics Model 2003; 21: 289 307.

40 Brooks B, Bruccoleri R, Olafson B, States D, Swaminathan S, Karplus M. CHARMM: A program for macromolecular energy, minimization, and dynamics calculations. J Comput Chem 1983; 4: 187-217.

41 Bartolott LJ, Flurchick K. (2007) An Introduction to Density Functional Theory, in Reviews in Computational Chemistry, Volume 7 (eds Lipkowitz KB and Boyd DB), John Wiley \& Sons, Inc, Hoboken, NJ, USA. doi: 10.1002/9780470125847.ch4.

42 Debnath AK. Generation of predictive pharmacophore models for CCR5 antagonists: study with piperidine- and piperazine-based compounds as a new class of HIV-1 entry inhibitors. J Med Chem 2003; 46: 4501-15.

43 Cokugras AN. Butyrylcholinestrase: structure and physiological importance. Turk J Biochem 2003; 28: 54-61.

44 Darvesh KV, McDonald RS, Mataija D, Walsh R, Mothana S, Lockridge 
O, et al. Carbamates with differential mechanism of inhibition toward acetylcholinesterase and butyrylcholinesterase. J Med Chem 2008; 51: 4200-12.

45 Li Y, Wang Y, Li W, Dong P, Ge G, Yang L, et al. Investigation of binding features: effects on the interaction between CYP2A6 and inhibitors. J
Comput Chem 2010; 31: 1822-31.

46 Queiroz AN, Gomes BAQ, Moraes WM Jr, Borges RS. A theoretical antioxidant pharmacophore for resveratrol. Eur J Med Chem 2009; 44: 1644-49. 